\title{
Zero-bias conductance peak in Dirac semimetal-superconductor devices
}

\author{
W. Yu, ${ }^{1, *}$ Rafael Haenel $\odot,{ }^{2, *}$ M. A. Rodriguez, ${ }^{1}$ S. R. Lee, ${ }^{1}$ F. Zhang $\odot,{ }^{3}$ M. Franz, ${ }^{2}$ D. I. Pikulin, ${ }^{4, \dagger}$ and W. Pan ${ }^{1,5, *}$ \\ ${ }^{1}$ Sandia National Laboratories, Albuquerque, New Mexico 87185, USA \\ ${ }^{2}$ Department of Physics and Astronomy, University of British Columbia, Vancouver, BC, Canada V6T 1Z1 \\ ${ }^{3}$ Department of Physics, University of Texas at Dallas, Dallas, Texas 75080, USA \\ ${ }^{4}$ Microsoft Quantum, Microsoft Station Q, University of California, Santa Barbara, California 93106, USA \\ ${ }^{5}$ Sandia National Laboratories, Livermore, California 94551, USA
}

(Received 16 September 2019; accepted 8 June 2020; published 1 July 2020)

\begin{abstract}
Majorana zero modes (MZMs), fundamental building blocks for realizing topological quantum computers, can appear at the interface between a superconductor and a topological material. One of the experimental signatures that has been widely pursued to confirm the existence of MZMs is the observation of a large, quantized zero-bias conductance peak (ZBCP) in the differential conductance measurements. In this Letter, we report observation of such a large ZBCP in junction structures of normal metal (titanium/gold Ti/Au)—Dirac semimetal (cadmiumarsenide $\mathrm{Cd}_{3} \mathrm{As}_{2}$ ) — conventional superconductor (aluminum $\mathrm{Al}$ ), with a value close to four times that of the normal state conductance. Our detailed analyses suggest that this large ZBCP is most likely not caused by MZMs. We attribute the ZBCP, instead, to the existence of a supercurrent between two far-separated superconducting $\mathrm{Al}$ electrodes, which shows up as a zero-bias peak because of the circuitry and thermal fluctuations of the supercurrent phase, a mechanism conceived by Ivanchenko and Zil'berman more than 50 years ago [Ivanchenko and Zilberman, JETP 28, 1272 (1969)]. Our results thus call for extreme caution when assigning the origin of a large ZBCP to MZMs in a multiterminal semiconductor or topological insulator/semimetal setup. We thus provide criteria for identifying when the ZBCP is definitely not caused by an MZM. Furthermore, we present several remarkable experimental results of a supercurrent effect occurring over unusually long distances and clean perfect Andreev reflection features.
\end{abstract}

DOI: 10.1103/PhysRevResearch.2.032002

Topological quantum computation (QC) [1,2] has emerged as a promising approach to QC due to its enhanced tolerance to errors which are induced by inevitable coupling to the environment. In this scheme, a quantum bit (qubit) would be constructed out of four Majorana quasiparticles (MQPs) [3], with a gate performed by moving one MQP around another, or braiding MQPs. Because the braiding is topological and nonlocal, Majorana-based qubits would be intrinsically robust against local decoherence sources and thereby enable faulttolerant QC.

Until 2008, the search for MQPs in solid-state systems focused on the exotic $5 / 2$ fractional quantum Hall state [4,5], for which the earlier theoretical framework of TQC was developed [2]. However, concrete evidence of such quantum Hall non-Abelian anyons has not been established. The advent of topological insulators has prompted proposals for realizing Majorana quasiparticles via the superconducting proximity effect $[6,7]$. In these systems, the Majorana quasiparticles are

\footnotetext{
*These authors contributed equally to this work.

†mpikuli@microsoft.com

‡wpan@sandia.gov
}

Published by the American Physical Society under the terms of the Creative Commons Attribution 4.0 International license. Further distribution of this work must maintain attribution to the author(s) and the published article's title, journal citation, and DOI. predicted to appear as topological defects, namely Majorana zero modes (MZMs), at the superconductor-magnet interface or $\pi$-Josephson junction at the surface of a topological insulator. Soon after this pioneering theoretical work, it was realized that MZMs could also be realized in a semiconductor system in combination with a strong spin-orbit coupling, an external magnetic field, and the superconducting proximity effect $[8-10]$. One of the experimental signatures that has been widely pursued to confirm the existence of MZMs is the observation of a large, quantized in units of $2 e^{2} / h$ zerobias conductance peak (ZBCP) in the differential conductance measurements. ZBCPs have been observed in InSb and InAs nanowire systems [11-17] and ferromagnetic atom chains [18]. However, the origin of the observed ZBCPs in these experiments remains debated [19-22]. As such, more experiments are imperative to establish MZMs in other topological quantum material systems beyond semiconducting nanowires. In the following, we will present results in a Dirac semimetal $[23,24]$ cadmium-arsenide $\left(\mathrm{Cd}_{3} \mathrm{As}_{2}\right)$ that show a large, nearly quantized in units of normal state conductance ZBCP in junction structures of normal metal (titanium/gold Ti/Au), $\mathrm{Cd}_{3} \mathrm{As}_{2}$, conventional superconductor (aluminum $\mathrm{Al}$ ). Our detailed analyses suggest that this large ZBCP is most likely not caused by MZMs. We attribute the ZBCP, instead, to the existence of a supercurrent between two far-separated superconducting $\mathrm{Al}$ electrodes, which shows up as a zero-bias peak because of the circuitry and thermal fluctuations of the supercurrent phase, a mechanism conceived by Ivanchenko 
(a)

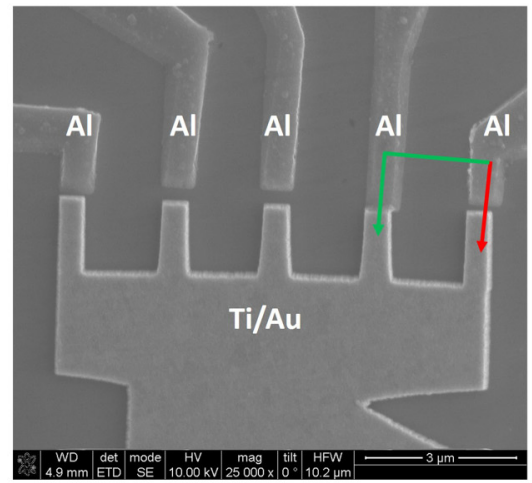

(c)

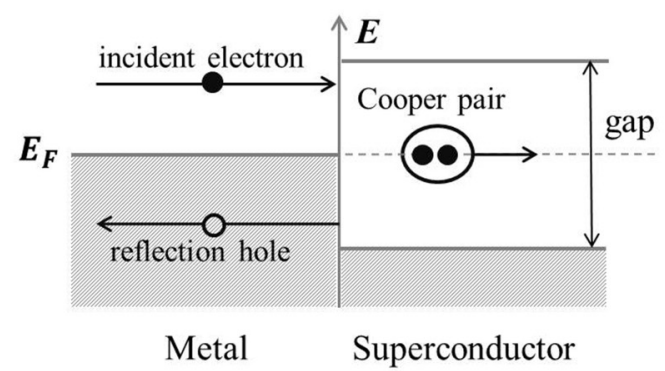

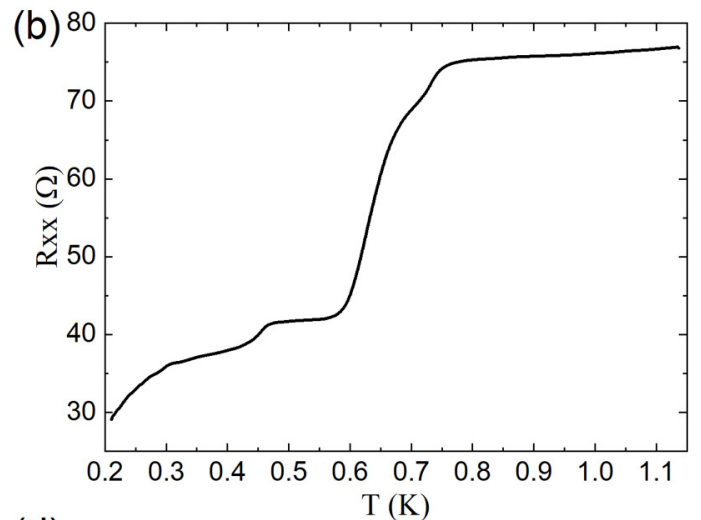

(d)

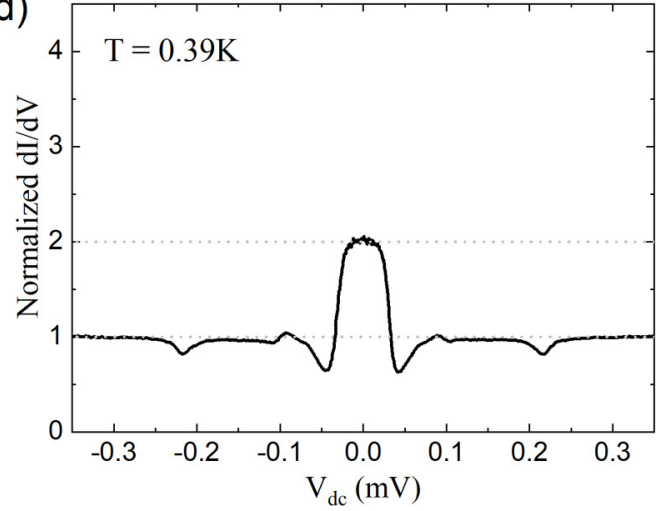

FIG. 1. (a) An SEM picture of the device. The Ti/Au and Al electrodes are deposited on the $\mathrm{Cd}_{3} \mathrm{As}_{2}$ thin flake. (b) Temperature dependence of the junction resistance. (c) A schematic diagram showing the Andreev reflection. (d) The normalized differential conductance $d I / d V$ versus the d.c. bias across the junction $\left(V_{\mathrm{dc}}\right)$ measured in our device at $T=0.39 \mathrm{~K}$ at zero magnetic field.

and Zil'berman more than 50 years ago [25-27]. Our results thus call for extreme caution when assigning the origin of a large $\mathrm{ZBCP}$ to $\mathrm{MZM}$ in a multiterminal semiconductor or topological insulator/semimetal setup. We thus provide criteria for identifying when the ZBCP is definitely not caused by an MZM.

We choose $\mathrm{Cd}_{3} \mathrm{As}_{2}$ in this study due to the following reasons. First, it has been shown that the proximitized Fermi arc surface states [24] in $\mathrm{Cd}_{3} \mathrm{As}_{2}$ can show properties of a topological superconductor [28-30]. The induced pairing in these topological superconducting surface states can give rise to topologically protected gapless Andreev bound states, i.e., Majorana zero modes. Second, to our knowledge, $\mathrm{Cd}_{3} \mathrm{As}_{2}$ is the only topological semimetal system where the evidence of Majorana flat bands [31] have been observed [28]. This provides high confidence that localized Majorana zero modes can also be realized in $\mathrm{Cd}_{3} \mathrm{As}_{2}$. Third, as will see below, the decoupling of the surface and bulk states in $\mathrm{Cd}_{3} \mathrm{As}_{2}$ enables the Josephson effect that gives rise to the large ZBCP over a distance much longer than the coherence length. Finally, $\mathrm{Cd}_{3} \mathrm{As}_{2}$ is air stable and less prone to the oxidization issue that has troubled other topological materials such as $\mathrm{ZrTe}_{5}$ and $\mathrm{Bi}_{2} \mathrm{Te}_{3}$. This makes $\mathrm{Cd}_{3} \mathrm{As}_{2}$ promising for practical quantum computation application. To search for MZMs, we follow the methodology developed in the InAs and InSb nanowire research [11-17] and fabricate $\mathrm{Ti} / \mathrm{Au}-\mathrm{Cd}_{3} \mathrm{As}_{2}-\mathrm{Al}$ junction structures. The mechanical exfoliation method is used to obtain the most flat and shiny $\mathrm{Cd}_{3} \mathrm{As}_{2}$ thin flakes for device fabrication from the initial ingot materials [28].
The $\mathrm{Cd}_{3} \mathrm{As}_{2}$ polycrystalline ingots used in this study are the same as those in Ref. [28]. The thickness of $\mathrm{Cd}_{3} \mathrm{As}_{2}$ flakes is about $200 \mathrm{~nm}$. To fabricate $\mathrm{Ti} / \mathrm{Au}-\mathrm{Cd}_{3} \mathrm{As}_{2}-\mathrm{Al}$ junctions, we deposit thin flakes of $\mathrm{Cd}_{3} \mathrm{As}_{2}$ on a $\mathrm{Si} / \mathrm{SiO}_{2}$ substrate followed by two-step electron beam lithography to define Ti/Au and Al electrodes, respectively. The thickness of the electrodes is $10 \mathrm{~nm} / 200 \mathrm{~nm}$ for Ti/Au and $300 \mathrm{~nm}$ for Al. A scanning electron microscopy image of one device is shown in Fig. 1(a). A total of five junctions are fabricated in this device and they share the same $\mathrm{Ti} / \mathrm{Au}$ electrode. The distance between two nearby junctions is $\sim 1.5 \mu \mathrm{m}$ in the device shown in Fig. 1(a), but is larger in other devices. In our experiment, only the right most junction is measured, and the four remaining $\mathrm{Al}$ contact leads are floating. The distance between the Ti/Au and $\mathrm{Al}$ electrodes is about $80 \mathrm{~nm}$. Figure 1(b) shows the temperature $(T)$ dependence of the resistance of this tunneling junction device. The superconducting transition temperature of $\mathrm{Al}$ electrode is at $\sim 1.2 \mathrm{~K}$ (not shown). The sharp drop at $T \sim 0.7 \mathrm{~K}$ is due to the onset of the proximity effect induced bulk superconductivity in $\mathrm{Cd}_{3} \mathrm{As}_{2}$.

Metal-superconductor junction device structures have been extensively utilized in the past for studying superconductivity [32]. Similar structures have also been used in studying excess conductance in two-dimensional electron gas in III-V semiconductors $[33,34]$. When such a device is biased above the superconducting gap, the device is in the normal state, the current $(I)$-voltage $(V)$ relationship is ohmic, and a constant differential conductance $(d I / d V)$ is measured. When the device bias is reduced and the incident electron from the normal 

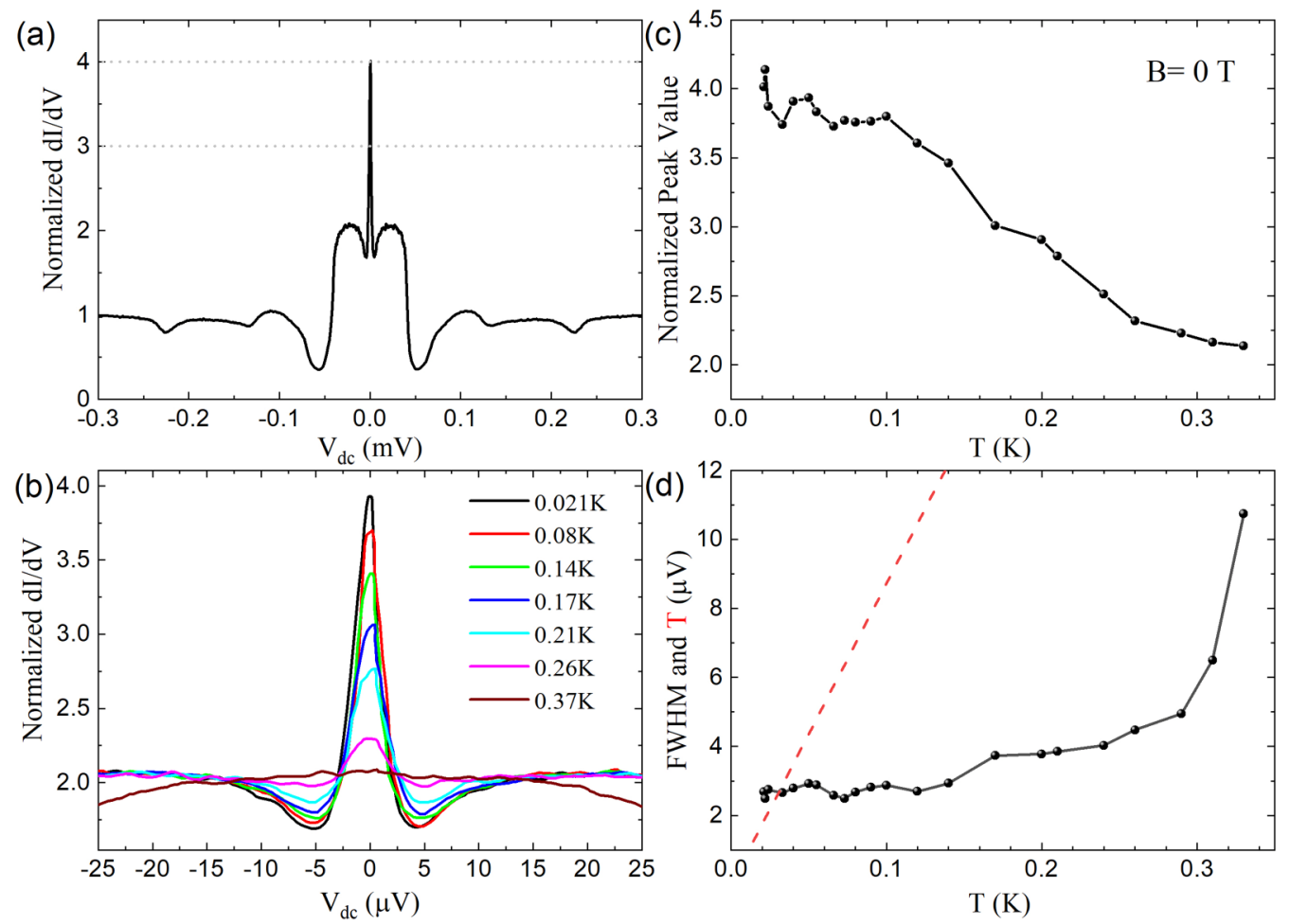

FIG. 2. (a) $d I / d V$ at base temperature $T=21 \mathrm{mK}$ at zero magnetic field. A zero-bias conductance peak (ZBCP) is observed. The value of ZBCP is four times of the normal state conductance $G_{N}$. (b) $d I / d V$ at various temperatures. (c) The peak value as a function of temperature. The value is nearly quantized at low temperatures and starts to decrease at $T$ is higher than $100 \mathrm{mK}$. (d) The full width at half magnitude of $\mathrm{ZBCP}$ as a function of temperature. The red dashed line indicates the junction temperature.

metal is within the superconducting energy gap, the electron can form a Cooper pair with another electron in the metal, and this Cooper pair then tunnels into the superconductor. Due to this process, a hole with the opposite spin and velocity but equal absolute momentum is reflected to the metal. This process, known as Andreev reflection (AR), is schematically depicted in Fig. 1(c). If the interface quality is high, the conductance within superconducting energy gap becomes twice the normal conductance [32], and this is commonly dubbed perfect AR (PAR). Figure 1(d) shows a clear example of PAR observed in our device at $T=0.39 \mathrm{~K}$, where the normalized differential conductance $d I / d V$ is plotted as a function of d.c. voltage bias $\left(V_{\mathrm{dc}}\right)$ across the junction, and normalization is defined relative to the normal state conductance, $\mathrm{G}_{\mathrm{N}} \approx$ $0.0128 \Omega^{-1}$. At high $\left|V_{\mathrm{dc}}\right|$, the device is in the normal state and the conductance is constant, as expected. As $\left|V_{\mathrm{dc}}\right|$ decreases, moving into the superconducting gap, the conductance assumes a value of $2 \mathrm{G}_{\mathrm{N}}$. One can argue that the observed PAR is a signature of Klein tunneling in the $\mathrm{Cd}_{3} \mathrm{As}_{2}$ surface state [35]. The observation of this PAR also demonstrates (1) the formation of a hard gap induced by the proximity effect and (2) the absence of backscattering in the normal region.

As the junction is further cooled down, a large $\mathrm{ZBCP}$ is observed. The normalized conductance at the base temperature of $T=21 \mathrm{mK}$ and zero magnetic field is shown in Fig. 2(a). Overall, the curve is very similar to that in Fig. 1(d), except at $V_{\mathrm{dc}}=0 \mathrm{~V}$, where a large, sharp peak is observed. Notably, the value of this peak is four times that of the normal conductance value; this near-integer increase most likely is not, in itself, of fundamental significance, as modeling and discussion below will ultimately demonstrate. To further examine the origin of this $\mathrm{ZBCP}$, its temperature dependence is mapped out. Figure 2(b) shows the $d I / d V$ curves as a function of $V_{\mathrm{dc}}$ at various temperatures. In Fig. 2(c), the value of ZBCP is plotted versus $T$. At low temperatures, the amplitude is almost constant around $4 \mathrm{G}_{\mathrm{N}}$. It then starts decreasing at $T>0.1 \mathrm{~K}$ and eventually disappears around $T \sim 0.4 \mathrm{~K}$. Lorentzian fitting to each temperature curve is performed, from which the full width at half maximum (FWHM) of the ZBCP is obtained. In Fig. 2(d), we show the resulting temperature dependence of the FWHM. It is nearly constant at $\sim 3 \mu \mathrm{V}$ in the low temperature regime, where the amplitude of $\mathrm{ZBCP}$ is also constant. The FWHM starts increasing above $0.1 \mathrm{~K}$, where the ZBCP amplitude also starts decreasing. The FWHM increases sharply for $T>0.3 \mathrm{~K}$.

Below, we try to explain the data by considering two alternative mechanisms for ZBCP formation: (1) the existence of MZMs at the interface between a superconductor and a quantum spin Hall insulator stack/Dirac semimetal [31,3638] and (2) a supercurrent mechanism that may give rise to the observed large ZBCP [25]. Our detailed theoretical modeling will show that the latter origin is the one most likely to apply.

For the first mechanism, it is known that Fermi arc surface states of a Dirac semimetal can be viewed equivalently as a momentum-space stack of many identical two-dimensional quantum spin Hall insulator (QSHI) layers [38]. In other words, according to the theoretical work [38], in the presence of momentum conservation our junction structure could be viewed as a collection of many identical copies of Ti/Au-2D QSHI-Al junction devices. At low temperatures, MZMs can 

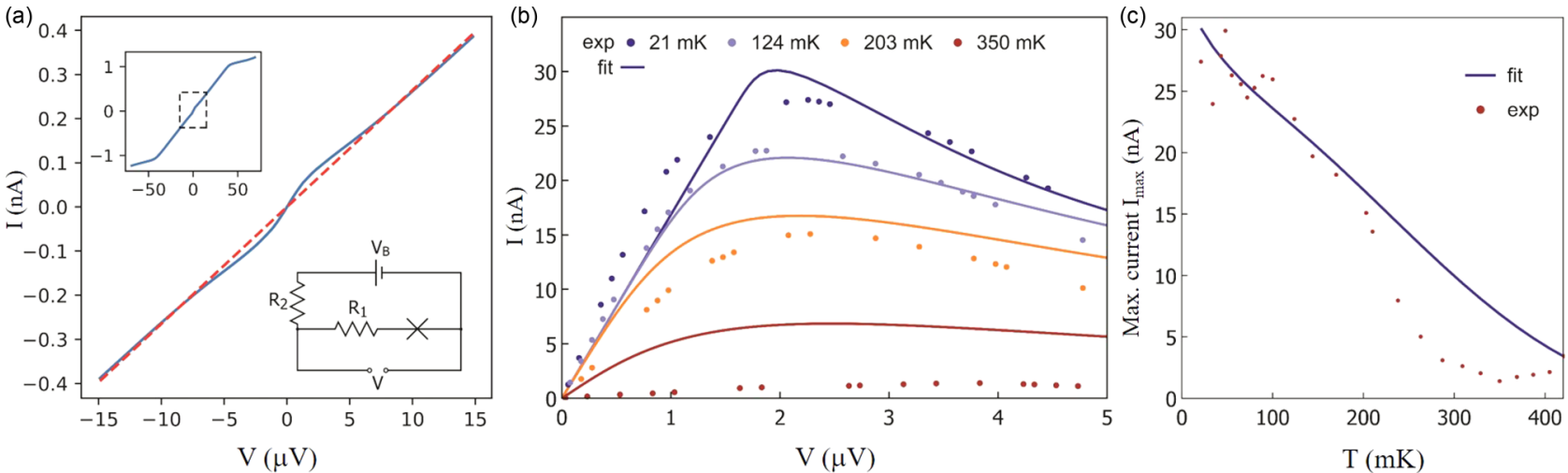

FIG. 3. (A) the $I-V$ curve at $T=21 \mathrm{mK}$, obtained by integrating the corresponding $d I / d V$ curve. Here only the data in the AR regime is shown. The red dashed line represents the contribution from AR. The up inset shows the whole I-V curve. The lower inset shows the effective circuit for the experimental setup. (B) IZ fitting at four selected temperatures. Dots are experimental data, line the IZ fitting. (C) The extracted maximal current at each temperature. The solid line is the theoretical fitting.

form at the interface between the QSHI edge channels and the superconductor [36,37], and in the presence of backscattering they contribute to a large, quantized in units of $2 N e^{2} / h \mathrm{ZBCP}$. Here $N$ is the number of channels in the junction.

However, our detailed examinations cast doubts over this mechanism. First, the width of ZBCP is too narrow, being even smaller than the measurement temperature [indicated by the red dashed line in Fig. 2(d)], while the width of MZM ZBCPs cannot be smaller than $3.5 K_{B} T$ [39]. This thus excludes a noninteracting electron explanation for the data. Second, magnetic backscattering is required to obtain localized MZMs that give rise to ZBCP. In the absence of magnetic scattering the weak antilocalization (WAL) effect in $\mathrm{Cd}_{3} \mathrm{As}_{2}$ should produce a zero-bias conductance dip [39]. This is inconsistent with the experimental observation.

Next, we consider the supercurrent mechanism and conduct an analysis in terms of an Resistively Shunted Junction (RSJ) model [26] in the presence of thermal fluctuations, which was solved by Ivanchenko and Zil'berman (IZ) [25]. First, where does the supercurrent in our setup arise? We assume good ohmic contact between the $\mathrm{Al}$ and $\mathrm{Ti} / \mathrm{Au}$ electrodes in each of the NS (normal metal-superconductor) junctions below the superconducting gap. Then two nearby Al electrodes, if they are phase coherent, can support supercurrents and additional current path (as shown by the green arrows in Fig. 1(a) besides the direct NS one [as shown by the red arrow in Fig. 1(a)] is available. Thermal fluctuations and circuitry move the maximum of the supercurrent from the zero bias. In this case the supercurrent peak in $I-V$ characteristics shows up a ZBCP in $d I / d V$. The large distance between the superconducting electrodes suppresses the supercurrent making the observed amplitude of ZBCP comparable to $G_{N}$.

In the following, we use the IZ formula [25] to quantitatively fit our measured differential conductance curves and extract parameters of our system. For this purpose, we first obtain $I-V$ curves by integrating the differential conductance. Figure 3(a) shows the result at $T=21 \mathrm{mK}$. The fitting range is limited within the Andreev reflection (AR) regime. Next, the linear contribution due to AR within the superconducting gap is subtracted from the $I-V$ curve as it is due to a parallel conductance channel. The obtained data represents the contribution from the supercurrent between two $\mathrm{Al}$ electrodes. The circuit diagram in the lower inset of Fig. 3(a) is an effective description for the experimental setup. Here, $V_{B}$ is the voltage source, $V$ the voltage that is plotted on the $x$ axis in Fig. 3(a), $R_{1}$ the junction resistance, and $R_{2}$ the lump resistance in the measurement circuit. We neglect the capacitance $C$ of the junction in the fitting. This is justified because of a large separation of Al electrodes.

The equations for the $I-V$ curve fitting are then given as follows [25]:

$$
\begin{aligned}
& I(V)=I_{0} \operatorname{Im}\left[\frac{I_{1-2 i \beta\left(V+R_{2} I\right) \hbar /\left(2 e\left(R_{1}+R_{2}\right)\right)}\left(\beta \frac{\hbar}{2 e} I_{0} \frac{\Delta(T)}{\Delta(0)}\right)}{I_{-2 i \beta\left(V+R_{2} I\right) \hbar /\left(2 e\left(R_{1}+R_{2}\right)\right)}\left(\beta \frac{\hbar}{2 e} I_{0} \frac{\Delta(T)}{\Delta(0)}\right)}\right], \\
& \frac{\Delta(T)}{\Delta(0)}=1-\sqrt{\frac{2 \pi k T}{\Delta(0)}} \exp \left(-\frac{\Delta(0)}{k T}\right) .
\end{aligned}
$$

Here, $I_{n}(x)$ is the modified Bessel function of complex order, $\Delta(0)=50 \mu \mathrm{eV}$ is the topological superconductivity gap at $T=0$, and $I_{0}$ the total critical current of the supercurrent quanta. The Bardeen-Cooper-Schrieffer temperature dependence of $\Delta(\mathrm{T}) / \Delta(0)$ is the first order expansion around $T=0$. In Figs. 3(b), we show the fitting at four selected temperatures (fitting at more temperatures can be found in the Supplemental Material [39]). It agrees well with the experimental data at low temperatures, e.g., at $T=21,124$, $203 \mathrm{mK}$. Fitting fails at higher temperatures, for example at $T=350 \mathrm{mK}$, where the temperature becomes comparable to the induced superconducting gap and quasiparticle contributions to current likely disturb the fitting. We note here that fitting using the phenomenological temperature dependence of $\Delta(T) / \Delta(0)=\tanh \left(1.74\left(T_{c} / T-1\right)^{1 / 2}\right)$ yields the same conclusion. In Fig. 3(c), the maximal current extracted from the fitting at each temperature is plotted.

The good agreement between the theoretical fitting and experimental data strongly supports that the ZBCP is due to the supercurrent between two separated $\mathrm{Al}$ electrodes. Moreover, because of this supercurrent origin, the FWHM width of $\mathrm{ZBCP}$ can be smaller than the measurement temperature, as 

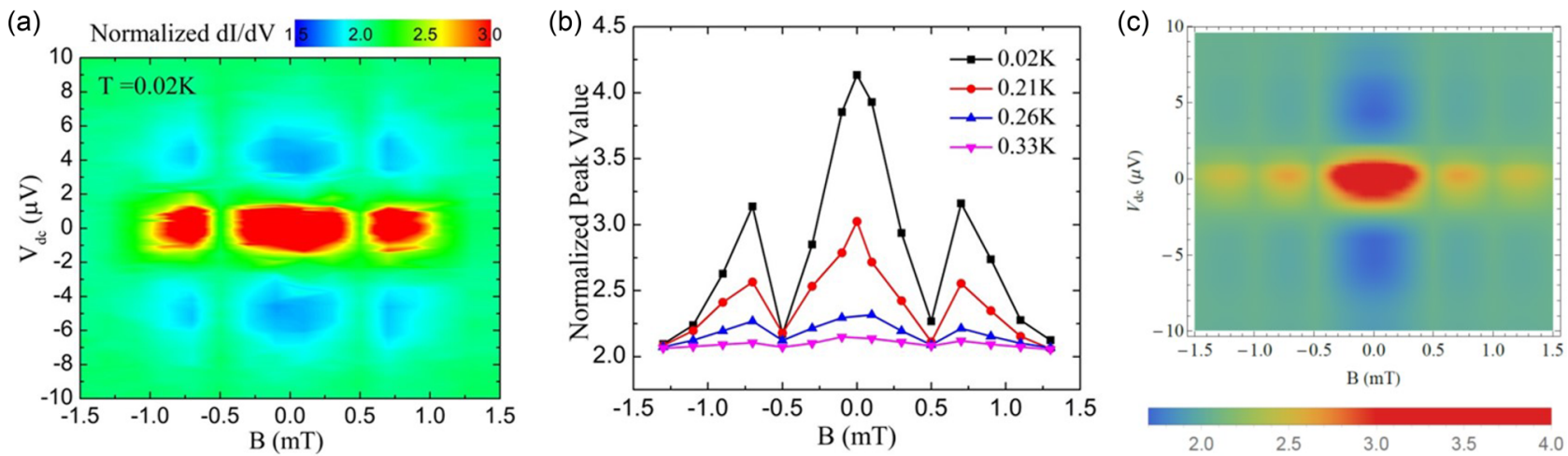

FIG. 4. (a) $2 \mathrm{D}$ color plot of $d I / d V$ as a function of magnetic field and $V_{\mathrm{dc}}$, respectively. ZBCP disappears at the magnetic field of $B \sim$ $0.5 \mathrm{mT}$ and for $B>1.3 \mathrm{mT}$. (b) The extracted peak value as a function of magnetic field at various temperatures. An oscillatory behavior is observed for the ZBCP value. (c) Theoretical simulation of the magnetic field dependence with a Fraunhofer pattern based on the experimental data at zero magnetic field. Good agreement between the experimental result (a) and theoretical simulation (c) is seen.

the width of the ZBCP is determined by the circuit parameters, not intrinsic temperature broadening. This explains the puzzle seen in Fig. 2(d).

The parameters obtained from the above fitting are $I_{0} \approx$ $35 \mathrm{nA}, R_{1} \approx 60 \Omega$, and $R_{2} \approx 90 \Omega . R_{1}$ is close to the normalstate resistance across the Ti/Au-Al junction, which is about $78 \Omega$. Based on these fitting parameters, we can estimate the number of spin-resolved supercurrent quanta in our device. First, we calculate the critical current $\Delta I_{0}$ of a single supercurrent quantum. It is given by $\Delta I_{0}=\Delta(0) \times \pi / e \times$ $G=6.1 \mathrm{nA}$ [40]. Here, $G=e^{2} / h$ for the helical (effectively spin-polarized) topological surface states of $\mathrm{Cd}_{3} \mathrm{As}_{2}$. Consequently, the total number of the supercurrent quanta is $34.8 / 6.1 \sim 6$.

Notably, the supercurrent mechanism also explains the peculiar magnetic $(B)$ field dependence of the ZBCP we observed. In Fig. 4 , the measured $d I / d V$ is plotted as a function of magnetic field and $V_{\mathrm{dc}}$, respectively. The ZBCP in our device is extremely sensitive to the magnetic fields and disappears at a magnetic field merely larger than $1.3 \mathrm{mT}$. Figure 4(b) shows the extracted experimental values of ZBCP at different temperatures demonstrating a clear oscillating pattern. For example, at the base $T$ of $21 \mathrm{mK}$, the amplitude decreases from $4 \mathrm{G}_{\mathrm{N}}$ at $B=0 \mathrm{~T}$ to $\sim 2 \mathrm{G}_{\mathrm{N}}$ at $0.5 \mathrm{mT}$. It then increases to $3 G_{N}$ at $0.75 \mathrm{mT}$, before it drops to zero again at higher magnetic fields. Since the critical magnet field of the superconducting $\mathrm{Al}$ electrodes is $\sim 40 \mathrm{mT}$, much larger than $1.3 \mathrm{mT}$, the disappearance cannot be due to the loss of the superconductivity in Al. Furthermore, this small period cannot be caused by the magnetic flux across the Ti/AuAl junction area. In fact, considering the area of the junction is $S \sim 4 \times 10^{-7} \mathrm{~m}^{2}$, magnetic flux quantum is inserted into the junction at $B=\frac{\varphi}{s}=52 \mathrm{mT}$, much larger than the observed $0.5 \mathrm{mT}$. Here $\varphi=2.067 \times 10^{-15} \mathrm{~Wb}$ is magnetic flux quantum. Thus, interference across this junction can be excluded as the source of the ZBCP oscillations. On the other hand, under the supercurrent model, the effective junction area should be the one between the two adjacent junction devices, which is about $4 \mu \mathrm{m}^{2}$. Consequently, the Fraunhofer period is $\sim 0.5 \mathrm{mT}$, consistent with the experimental observation.
Finally, one remark is in order before we conclude. The IZ model was conventionally used for short Josephson junctions. In our case, however, it still applies to a device where the two $\mathrm{Al}$ electrodes are separated by $\sim 10 \mu \mathrm{m}$, as shown in Fig. 5(a). In this device, the normal metal contacts are made of $\mathrm{Ni} / \mathrm{Au}$. The differential conductance between the Ni/Au and $\mathrm{Al}$ electrodes for the middle junction is shown in Fig. 5(b). Overall, it is similar to that in the first device already discussed, with both exhibiting a perfect AR and a large ZBCP. Applying the IZ fitting yields again a good agreement, as shown in Fig. 5(c) for the 98-mK data and in Fig. 5(d) for the extracted $I_{0}$. However, unlike the first specimen where the separation between two adjacent junctions is comparable to the $\mathrm{Al}$ superconducting coherence length $\left(\xi_{\mathrm{Al}} \sim 1 \mu \mathrm{m}\right)$, $\mathrm{Cd}_{3} \mathrm{As}_{2}$ phase coherence length $\left(l_{\phi} \sim 0.6 \mu \mathrm{m}\right)[40]$, and/or $\mathrm{Cd}_{3} \mathrm{As}_{2}$ thermal length $L_{T}$ (at $T=20 \mathrm{mK}, L_{T} \sim 1.1 \mu \mathrm{m}$ is estimated using a diffusion constant of $D \sim 0.02 \mathrm{~m}^{2} / \mathrm{s}$ [41]), the separation of $\mathrm{Al}$ electrodes in this device is now $\sim 10 \mu \mathrm{m}$, much longer than $\xi_{\mathrm{Al}}$ and $l_{\phi}$. Thus, it is totally surprising that the supercurrent appears to still exist over such a large distance. In the following, we discuss three possibilities to resolve this apparent difficulty in our interpretation. First, $l_{\phi}$ entering the WAL measurements [42] is different from $l_{\phi}$ relevant for the Aharonov-Bohm-like interference necessary for the Fraunhofer pattern [43]. Second, the supercurrent is proportional to the transmission (to the power of 2) through the channels connecting the superconductors. In our device with large $\mathrm{Al}$ electrodes, there can exist a tail of the channel transmission distribution responsible for the supercurrent. Third, it is possible that existence of supercurrent is determined by the electron pairing coherence length, which might be very long in the topological surface states in $\mathrm{Cd}_{3} \mathrm{As}_{2}$ due to the suppressed backscattering. Finally, the Josephson effect over such a long distance seems to indicate relative decoupling of the surface and bulk states, which can be used for potential applications of the material.

In summary, we report observation of a large ZBCP in tunnel junctions made of normal metal (Ti/Au)-Dirac semimetal $\left(\mathrm{Cd}_{3} \mathrm{As}_{2}\right)$-conventional superconductor $(\mathrm{Al})$. Our detailed analyses suggest that this large ZBCP probably is due to the existence of a supercurrent between two far-separated 

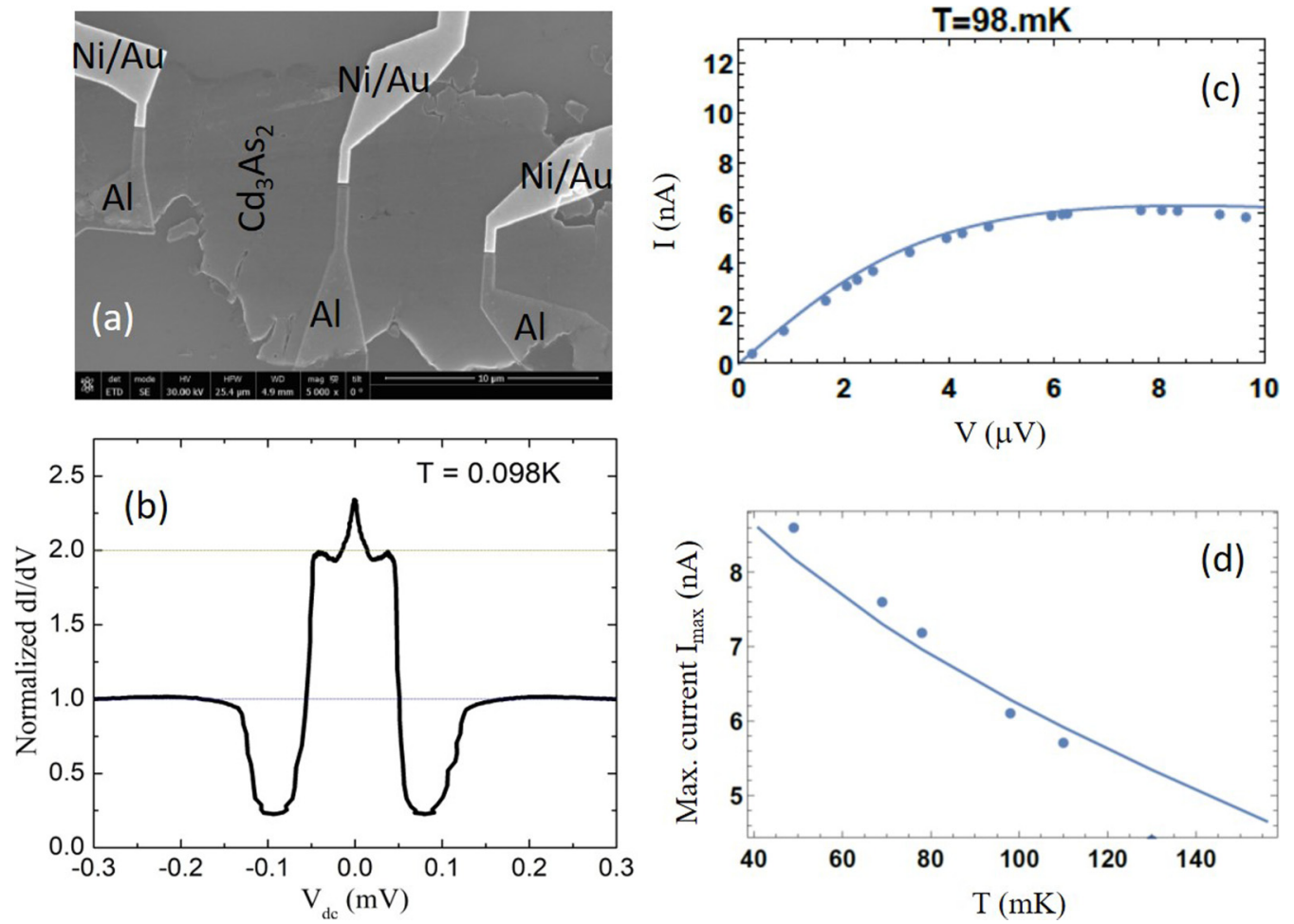

FIG. 5. (a) SEM image of another device where the tunnel junctions are separated by $\sim 10 \mu \mathrm{m}$. The irregular shaped $\mathrm{Cd}_{3} \mathrm{As}_{2}$ thin flake is visible. (b) Large ZBCP measured in the middle junction at $T=98 \mathrm{mK}$. (c) IZ fitting to the $T=98 \mathrm{mK}$ data. (d) $I_{0}$ as a function of temperature. The solid line is the theoretical fitting.

superconducting $\mathrm{Al}$ electrodes. Our results thus call for extreme caution when assigning a large ZBCP to the MZM origin, especially when the width of the $\mathrm{ZBCP}$ is below $3.5 K_{B} T$

The work at Sandia National Labs was supported by a Laboratory Directed Research and Development project. Device fabrication was performed at the Center for Integrated Nanotechnologies, an Office of Science User Facility operated for the U.S. Department of Energy (DOE) Office of Science. Sandia National Laboratories is a multimission laboratory managed and operated by National
Technology \& Engineering Solutions of Sandia, LLC, a wholly owned subsidiary of Honeywell International Inc., for the U.S. Department of Energy's National Nuclear Security Administration under Contract No. DE-NA0003525. This paper describes objective technical results and analysis. Any subjective views or opinions that might be expressed in the paper do not necessarily represent the views of the U.S. Department of Energy or the United States Government. Work at UT Dallas was supported by ARO under Grant No. W911NF-18-1-0416 and NSF under Grant No. DMR-1921581. Work at UBC was supported by NSERC and CIFAR.
[1] A. Yu. Kitaev, Ann. Phys. 303, 2 (2003).

[2] C. Nayak, S. H. Simon, A. Stern, M. Freedman, and S. Das Sarma, Rev. Mod. Phys. 80, 1083 (2008).

[3] S. R. Elliott and M. Franz, Rev. Mod. Phys. 87, 137 (2015).

[4] R. Willett, J. P. Eisenstein, H. L. Störmer, D. C. Tsui, A. C. Gossard, and J. H. English, Phys. Rev. Lett. 59, 1776 (1987).

[5] W. Pan, J.-S. Xia, V. Shvarts, D. E. Adams, H. L. Stormer, D. C. Tsui, L. N. Pfeiffer, K. W. Baldwin, and K. W. West, Phys. Rev. Lett. 83, 3530 (1999).

[6] L. Fu and C. L. Kane, Phys. Rev. Lett. 100, 096407 (2008).

[7] M. Franz, Nat. Nanotech. 8, 149 (2013).

[8] R. M. Lutchyn, J. D. Sau, and S. Das Sarma, Phys. Rev. Lett. 105, 077001 (2010).

[9] Y. Oreg, G. Refael, and F. von Oppen, Phys. Rev. Lett. 105, 177002 (2010).
[10] J. Alicea, Phys. Rev. B 81, 125318 (2010).

[11] K. Mourik, K. Zuo, S. M. Frolov, S. R. Plissard, E. P. A. M. Bakkers, and L. P. Kouwenhoven, Science 336, 1003 (2012).

[12] L. P. Rokhinson, X. Liu, and J. K. Furdyna, Nat. Phys. 8, 795 (2012).

[13] A. Das, Y. Ronen, Y. Most, Y. Oreg, M. Heiblum, and H. Shtrikman, Nat. Phys. 8, 887 (2012).

[14] M. T. Deng, C. L. Yu, G. Y. Huang, M. Larsson, P. Caroff, and H. Q. Xu, Nano Lett. 12, 6414 (2012).

[15] A. D. K. Finck, D. J. Van Harlingen, P. K. Mohseni, K. Jung, and X. Li, Phys. Rev. Lett. 110, 126406 (2013).

[16] M. T. Deng, S. Vaitiekenas, E. B. Hansen, J. Danon, M. Leijnse, K. Flensberg, J. Nygård, P. Krogstrup, and C. M. Marcus, Science 354, 1557 (2016). 
[17] H. Zhang, C.-X. Liu, S. Gazibegovic, D. Xu, J. A. Logan, W. Wang, N. van Loo, J. D. S. Bommer, M. W. A. de Moor, D. Car, R. L. Op het Veld, P. J. van Veldhoven, S. Koelling, M. A. Verheijen, M. Pendharkar, D. J. Pennachio, B. Shojaei, J. S. Lee, C. J. Palmstrøm, E. P. A. M. Bakkers, S. Das Sarma, and L. P. Kouwenhoven, Nature (London) 556, 74 (2018).

[18] S. Nadj-Perge, I. K. Drozdov, J. Li, H. Chen, S. Jeon, J. Seo, A. H. MacDonald, B. A. Bernevig, and A. Yazdani, Science 346, 602 (2014).

[19] K. T. Law, P. A. Lee, and T. K. Ng, Phys. Rev. Lett. 103, 237001 (2009).

[20] C.-X. Liu, J. D. Sau, T. D. Stanescu, and S. Das Sarma, Phys. Rev. B 96, 075161 (2017).

[21] C. Moore, C. Zeng, T. D. Stanescu, and S. Tewari, Phys. Rev. B 98, 155314 (2018).

[22] O. A. Awoga, J. Cayao, and A. M. Black-Schaffer, Phys. Rev. Lett. 123, 117001 (2019).

[23] S. M. Young, S. Zaheer, J. C. Y. Teo, C. L. Kane, E. J. Mele, and A. M. Rappe, Phys. Rev. Lett. 108, 140405 (2012).

[24] Z. Wang, H. Weng, Q. Wu, X. Dai, and Z. Fang, Phys. Rev. B 88, 125427 (2013).

[25] Yu. M. Ivanchenko and L. A. Zil'berman, JETP 28, 1272 (1969).

[26] Michael Tinkham, Introduction to Superconductivity (Courier Corp., Chelmsford, MA, 2004).

[27] R. L. Kautz and John M. Martinis, Phys. Rev. B 42, 9903 (1990).

[28] W. Yu, W. Pan, D. L. Medlin, M. A. Rodriguez, S. R. Lee, Z.-Q. Bao, and F. Zhang, Phys. Rev. Lett. 120, 177704 (2018).
[29] A.-Q. Wang, C.-Z. Li, C. Li, Z.-M. Liao, A. Brinkman, and D.-P. Yu, Phys. Rev. Lett. 121, 237701 (2018).

[30] F. Zhang and W. Pan, Nat. Mater. 17, 851 (2018).

[31] A. Chen, D. I. Pikulin, and M. Franz, Phys. Rev. B 95, 174505 (2017).

[32] G. E. Blonder, M. Tinkham, and T. M. Klapwijk, Rev. Rev. B 25, 4515 (1982).

[33] A. Kastalsky, A. W. Kleinsasser, L. H. Greene, R. Bhat, F. P. Milliken, and J. P. Harbison, Phys. Rev. Lett. 67, 3026 (1991).

[34] F. Rahman, T. J. Thornton, R. Huber, L. F. Cohen, W. T. Yuen, and R. A. Stradling, Phys. Rev. B 54, 14026 (1996).

[35] S. Lee, V. Stanev, X. Zhang, D. Stasak, J. Flowers, J. S. Higgins, S. Dai, T. Blum, X. Pan, V. M. Yakovenko, J. Paglione, R. L. Greene, V. Galitski, and I. Takeuchi, Nature (London) 570, 344 (2019).

[36] L. Fu and C. L. Kane, Phys. Rev. B 79, 161408(R) (2009).

[37] H. Wang, H. C. Wang, H. W. Liu, H. Lu, W. H. Yang, S. Jia, X.-J. Liu, X. C. Xie, J. Wei, and J. Wang, Nat. Mater. 15, 38 (2016).

[38] A. Chen and M. Franz, Phys. Rev. B 93, 201105(R) (2016).

[39] See Supplemental Materials at http://link.aps.org/supplemental/ 10.1103/PhysRevResearch.2.032002 for theoretical model, some raw data, and data fitting code.

[40] N. M. Chtchelkatchev, G. B. Lesovik, and G. Blatter, Phys. Rev. B 62, 3559 (2000).

[41] M. N. Chernodub and M. A. Zubkov, Phys. Rev. B 95, 115410 (2017).

[42] B. Zhao, P. Cheng, H. Pan, S. Zhang, B. Wang, G. Wang, F. X. Xiu, and F. Q. Song, Sci. Rep. 6, 22377 (2016).

[43] T. Ludwig and A. D. Mirlin, Phys. Rev. B 69, 193306 (2004). 